\title{
Ginseng Extract Regulates the Alterations of Sleep Architecture and EEG Power Spectra in Restraint Stressed Rats
}

\author{
Yuan MA ${ }^{1}$, Jae Soon EUN ${ }^{2}$, Shulong YANG ${ }^{3}$, Kwang Seung $\mathrm{LEE}^{4}$, \\ Eun-Sil LEE ${ }^{4}$, Chung-Soo Kim ${ }^{5}$ and Ki-Wan $\mathrm{OH}^{5 *}$ \\ ${ }^{1}$ Beijing Institute of Pharmacology \& Toxicology, Beijing,100850, China. \\ ${ }^{2}$ College of Pharmacy, Woosuk University, Samrye, 565-701, Republic of Korea \\ ${ }^{3}$ Research Institute Veterinary Medicine, Chungbuk National University, Cheongju, 361-763, Republic of Korea \\ ${ }^{4}$ Research Institute of Nonghyup Korea Ginseng, Jeungpyung, 368-811, Republic of Korea \\ ${ }^{5}$ College of Pharmacy, Chungbuk National University, Cheongju, 361-763, Republic of Korea \\ (Received December 31, 2009; Revised March 5, 2010; Accepted March 8, 2010)
}

\begin{abstract}
The present investigation was conducted to evaluate the regulation of sleep architecture by the red ginseng water extract (RGE) in acutely and chronically restraint stressed rats. Adult rats were fitted with sleep-wake recording electrodes. Following post-surgical recovery, rats were extensively habituated for freely moving polygraphic recording conditions. Polygraphic signs of sleep-wake activities were recorded for $24 \mathrm{~h}$ after RGE administration and induction of stress and were analyzed to understand the regulation of sleep architecture. Acute stress decreased wakefulness and increased total sleep, non-rapid eye movement (NREM) sleep, and rapid eye movement (REM) sleep in both the daytime and nighttime recording. RGE shortened the daytime NREM and REM sleep, without changing the wakefulness and total sleep. RGE increased nighttime wakefulness, and decreased total, NREM and REM sleep. Chronic stress increased wakefulness and decreased total sleep in the daytime recording, and increased REM and decreased NREM sleep in both the day and night time recording. RGE ameliorated chronic stress and induced alterations of REM and NREM sleep in the day and night time sleep architecture. Acute and chronic stress could also induce alternations in cortex electroencephalogram (EEG) recording during NREM, REM sleep and wakefulness. These findings suggest that RGE may modulate the sleep behavior in acutely and chronically stressed rats and the ameliorating effect of RGE on the sleep architecture may involve in modulation of $\alpha-, \theta$ - and $\delta$ - wave activities of the cortical EEG.
\end{abstract}

Key words : electroencephalogram (EEG), power density, red ginseng, sleep, stress

\section{INTRODUCTION}

Ginseng as a traditional medicinal herb has been used to treat psychiatric disorders such as stress and insomnia for thousands of years in Asian countries. However, ginseng extract has been reported to have stimulant effects on the central nervous systems of humans and animals, and some patients experience CNS excitation, arousal and sleeplessness [1, 2]. Moreover, ginseng has been reported to induce insomnia, nervousness and diarrhea after long term use as well as when it is taken at higher dosages. Nevertheless, it has been said to possess neuroprotective effects anti-stress activity and has been promoted as a tonic that is capable of invigorating the physical and

* Corresponding author. E-mail: kiwan@chungbuk.ac.kr Phone: +82-43-261-2827, Fax: +82-43-261-2827 mental well-being [3-7]. Ginseng serves as an "adaptogen", a term used to describe a chemical that increases the resistance to stresses. This adaptogenic quality, however, is thought to be secondary to the normalization of body processes through the regulation of the production of various hormones. Ginseng stabilizes and balances physiology, and may help to maintain normal sleep and wakefulness [8]. In previous research, red ginseng water extract (RGE) decreased the power density of cortical electroencephalogram (EEG) $\delta$ waves $(0.75-4.5 \mathrm{~Hz})$ and increased $\alpha$-waves (8.0-13.0 Hz) in non-rapid eye movement (NREM) and rapid eye movement (REM) sleep. It also decreased $\delta$-wave power density during wakefulness. However, RGE increased spontaneous sleep and NREM sleep $[9,10]$.

In addition, there has been increased interest in whether RGE regulates the alteration of the sleep architecture and the EEG power spectra in acutely and chronically stressed 
rats. Growing evidence indicates a close relationship between the stress response and the resultant sleep pattern [11]. This notable alternation of sleep after stress suggests the participation of neurotransmitters and hormones [12-14]. Moreover, chronic stress produces sleep disturbances characterized by a chronic immobilization stress decrease in active waking, NREM and REM sleep, a blunting of the sleep-wake cycle, and a decrease in REM sleep [15]. This sleep-disturbance is usually viewed as one of the deleterious effects that stress has on the CNS [16]. The purpose of this study was to evaluate whether RGE ameliorates the sleep disrupting actions of stress using a rat immobilization model.

\section{MATERIALS AND METHODS}

\section{Preparation of red ginseng water extract (RGE)}

Red ginseng water extract (RGE) was kindly provided by Nonghyup Korea Ginseng (Jeungpyung, Korea). Ginsenosides, the major components of RGE were standardized by Nonghyup Co. Ltd., and the saponin fraction yield from RGE was $4.5 \%$.

\section{Experimental animals}

Experiments were performed on 80 adult male Wistar rats (Samtako, Osan, Korea) weighing between 250 and $350 \mathrm{~g}$. The rats were housed individually with food, and water was provided ad libitum under an artificial $12 \mathrm{~h}$ light/dark cycle (light on at 7:00) and at a constant temperature $\left(22 \pm 2^{\circ} \mathrm{C}\right)$. The rats were housed in the departmental holding room for 1 week before testing. All the rats were maintained, and all experiments were conducted, according to the guide for the Care and Use of Laboratory Animals (National Academic Press, Washington, DC, 1996), and in accordance with the National Institute of Toxicological Research on the Korea Food and Drug Administration guideline for the care and use of laboratory animals.

\section{Surgery}

All surgical procedures were performed stereotaxically under aseptic conditions. Surgical anesthesia was achieved with pentobarbital (50 mg/kg, ip) and all efforts were made to minimize the suffering of the animals. Each rat was implanted with a transmitter (Data Sciences International TA11CTA-F40, MN, USA) for recording EEG and activity via telemetry as described previously [17]. The body of the transmitter was implanted subcutaneously off midline and posterior to the scapula and was attached to the skin with 3 sutures for stabilization. Leads from the transmitter were led subcutaneously to the skull and the bare ends were placed in contact with the dura through holes that were made in the skull (A: 2.0 [Bregma], L: 1.5; P: 7.0 [Bregma], L: 1.5 contra-lateral). The electrodes were anchored to the skull with screws and dental cement.

\section{Experiment procedure}

Following 7 days post-surgical recovery, rats were divided into control (non-stress), acute stress, and chronic stress groups, with 8 rats in each group; the RGE-treated groups were also divided into an acute stress and chronic stress groups, with 8 rats in each group. RGE were dissolved in distilled water and administered orally $60 \mathrm{~min}$ before the application of the restraint stress. Rats in the acute stress RGE group were fed with RGE 25, 50 and $100 \mathrm{mg} / \mathrm{kg}$ for 10 days. On the $8^{\text {th }}, 9^{\text {th }}$ and $10^{\text {th }}$ day of RGE feeding, the rats were stressed for $3 \mathrm{~h}$ once per day. In the chronic stress RGE group, rats were fed with RGE 25,50 and $100 \mathrm{mg} / \mathrm{kg}$ for 10 days. On the $10^{\text {th }}$ day and 1 $\mathrm{h}$ after RGE feeding, the rats were once stressed for $22 \mathrm{~h}$. One hour after ending the stress procedure on the $10^{\text {th }}$ day in both of acute and chronic stress groups, sleeping behavior and EEG data were initiated and continuously recorded for $24 \mathrm{~h}(12 \mathrm{~h}$ day time and $12 \mathrm{~h}$ night time recording). The total quantity of RGE consumption for each rats in 25, 50 and $100 \mathrm{mg} / \mathrm{kg}$ RGE administration group were $62.5,125$ and $250 \mathrm{mg}$ respectively. Briefly, for acute stress group, rats were fed with RGE at 2:00 pm every day for 10 days. On the 8th, 9th and 10th day of RGE feeding, the rats were stressed for $3 \mathrm{~h}$ once from 3:00 pm to 6:00 pm per day; for chronic stress group, rats were fed with RGE at 2:00 pm every day for 9 days, on the 10th day RGE were fed at 7:00 pm, $1 \mathrm{~h}$ later, chronic restraint were loaded from 8:00 pm to next day 6:00 pm. Sleep behaviors and EEG were recorded $1 \mathrm{~h}$ after the finish of the last acute or chronic restraint, from 7:00 pm and lasted for $24 \mathrm{~h}$.

The stress was produced by restraining the animal inside an adjustable acrylic hemi-cylindrical plastic tube (7.5-cm diameter, $15-\mathrm{cm}$-long) individually. In order to induce a response of combined or mixed stress, which means rats experienced physical, psychological/emotional and other stressors in restraint process, the stressed rats could not eat food or drink water under restraint process $[18,19]$. All experiments were conducted according to the guide for the Care and Use of Laboratory Animals (National Academic Press, Washington, DC, 1996), and in accordance with the National Institute of Toxicological 
Research on the Korea Food and Drug Administration guideline for the care and use of laboratory animals.

\section{Data Collection}

Telemetric recording of cortical EEG and activity were conducted using procedures similar to previous reports [17]. For the EEG signal, the gain of transmitters was set at $0.5 /+0.5$ volts per/units $\times 2$ and the raw signals generated from the transmitter were in the range of $0.5-20.0 \mathrm{~Hz}$. The signals were processed by a Data Sciences International analog converter and routed to an $\mathrm{AD}$ converter (Eagle PC30, USA) housed in a PC class computer. The AD converter digitized the EEG and activity signals at $128 \mathrm{~Hz}$. The digitized data were transferred to the computer and displayed graphically by the program on the computer monitor. An on-line fast Fourier transformation (FFT) was performed on the EEG data at $2 \mathrm{sec}$ intervals during data acquisition (256 samples) after a Hanning window treatment. The FFT analysis generated power density values from 0.0 to $20.0 \mathrm{~Hz}$ at a resolution of $0.5 \mathrm{~Hz}$. The FFT data were further averaged in the range of 0 to $20 \mathrm{~Hz}$ for every $10 \mathrm{sec}$. The sleep data and FFT results were saved to the hard disk every $10 \mathrm{sec}$ for additional off-line analysis. Movement of the animal in relation to the telemetry receiver generated transistor-transistor logic (TTL) pulses that were collected and counted as a measure of activity. Oral administration of RGE was loaded $10 \mathrm{~min}$ before the EEG recording. Recording began at 7:00 am and the $24 \mathrm{~h}$ EEG and activity were recorded in each rat.

\section{Determination of behavioral states and analysis in} EEG power

The amount of time in wakefulness, NREM and REM sleep were determined from the digitized data at $10 \mathrm{sec}$ intervals using professional animal sleep analysis software SleepSign 2.1 (KISSEI Comtec Co Ltd, Matsumoto, Japan). Briefly, the software discriminates wakefulness as high-frequency low-amplitude EEG. NREM was scored based on the presence of spindles interspersed with slow waves in the EEG. EEG power during REM is significantly reduced in lower frequency $\delta$-wave $(0.75-4.0 \mathrm{~Hz})$ and increased in the range of $\theta$-wave activity $(5.0-9.0 \mathrm{~Hz}$, peak at $7.5 \mathrm{~Hz}$ ).

\section{Data analysis}

The time spent (min) in NREM, REM, total sleep time (NREM+REM) and numbers of sleep-wake cycle were processed to obtain $12 \mathrm{~h}$ period totals for each rat. We further calculated the time of each recording spent in each sleep-wake state (wake, NREM, REM). The absolute EEG power during wakefulness, NREM, and REM were calculated in $0.5 \mathrm{~Hz}$ bins from 0.5 to $20 \mathrm{~Hz}$ for the entire $12 \mathrm{~h}$ reading of each recording process. EEG power density in 3 selected frequency bands for wakefulness, NREM, and REM ( $\delta$-wave $(0.75-4.5 \mathrm{~Hz}$ ), $\theta$-wave (5.0-9.0 $\mathrm{Hz}$ ) and $\alpha$-wave (8.0-13.0 Hz)) were subsequently evaluated.

\section{Statistical analysis}

All statistical analyses were conducted using SigmaStat software. Measures of analysis of variance (ANOVA) procedures were used in the data analysis. After extensive ANOVA testing, post hoc comparisons were conducted using the Tukey's test.

\section{RESULTS}

\section{Effects of RGE on sleep architecture of acutely stressed rats}

Fig. 1 shows the effect of RGE on the sleep architecture of acutely stressed rats. The ANOVAs were significant for comparisons across groups in time spent in wakefulness, total sleep, NREM and REM sleep of acutely stressed rats in day time recording (Wakefulness $[\mathrm{F}(4,35)=3.91, p<$ $0.01]$; Total sleep $[\mathrm{F}(4,35)=3.91, p<0.01]$; NREM sleep $[\mathrm{F}(4,35)=3.93, \quad p<0.01] ; \quad \mathrm{REM}$ sleep $[\mathrm{F}(4,35)=2.82$, $p<0.05])$ and night time recording (Wakefulness $[\mathrm{F}(4,35)$ $=3.98, p<0.01]$; Total sleep $[\mathrm{F}(4,35)=3.98, p<0.01]$; NREM sleep $[\mathrm{F}(4,35)=2.63, p<0.05]$; REM sleep $[\mathrm{F}(4,35)=2.92$, $p<0.05])$.

Post hoc comparisons revealed that acute stress decreased wakefulness, increased total, NREM, and REM sleep in both daytime and night time recording. In the day time recording, NREM and REM sleep in stressed rats were shortened after administration of RGE at both the 25 and the $100 \mathrm{~g} / \mathrm{kg}$ dose with no effect on the time of wakefulness and total sleep ( $p<0.05, p<0.01$, Fig. 1A). RGE, at 25 and $50 \mathrm{~g} / \mathrm{kg}$ doses, significantly ameliorated the stress induced alterations in the night time sleep architecture, increased wakefulness, and decreased total, NREM and REM sleep. At $100 \mathrm{~g} / \mathrm{kg}$, RGE decreased REM sleep only $(p<0.05, p<0.01$, Fig. 1B).

\section{Effects of RGE on sleep/wake changes of acutely stressed rats}

Fig. 2 presents the effect of RGE on sleep/wake changes in acutely stressed rats. The ANOVAs were significant for comparisons across groups in sleep/wake changes of 


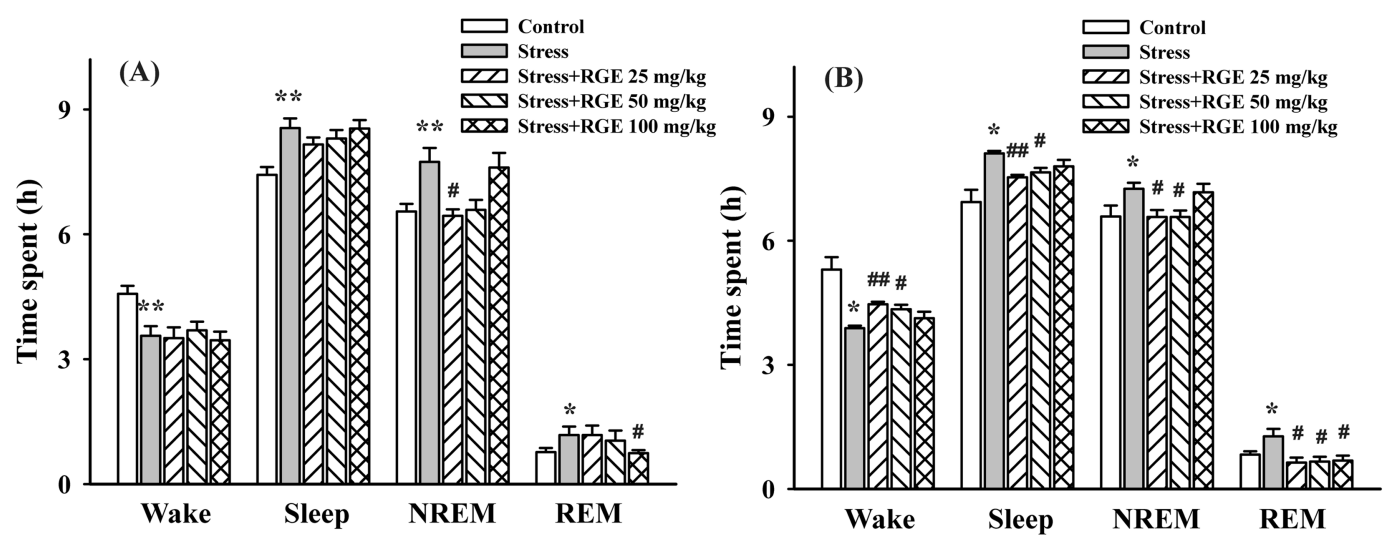

Fig. 1. The effect of RGE on sleep architecture in acutely stressed rats. The data represent the mean \pm SE of time spent in sleep-wake state [Wake, Sleep (total sleep)], NREM sleep, REM sleep). (A): Day time sleep architecture in acutely stressed rats. (B): Night time sleep architecture in acutely stressed rats. ${ }^{*} \mathrm{P}<0.05$; $* * \mathrm{P}<0.01$, significantly different from control group; \#P<0.05; $\# \# \mathrm{P}<0.01$, significantly different from stress group.
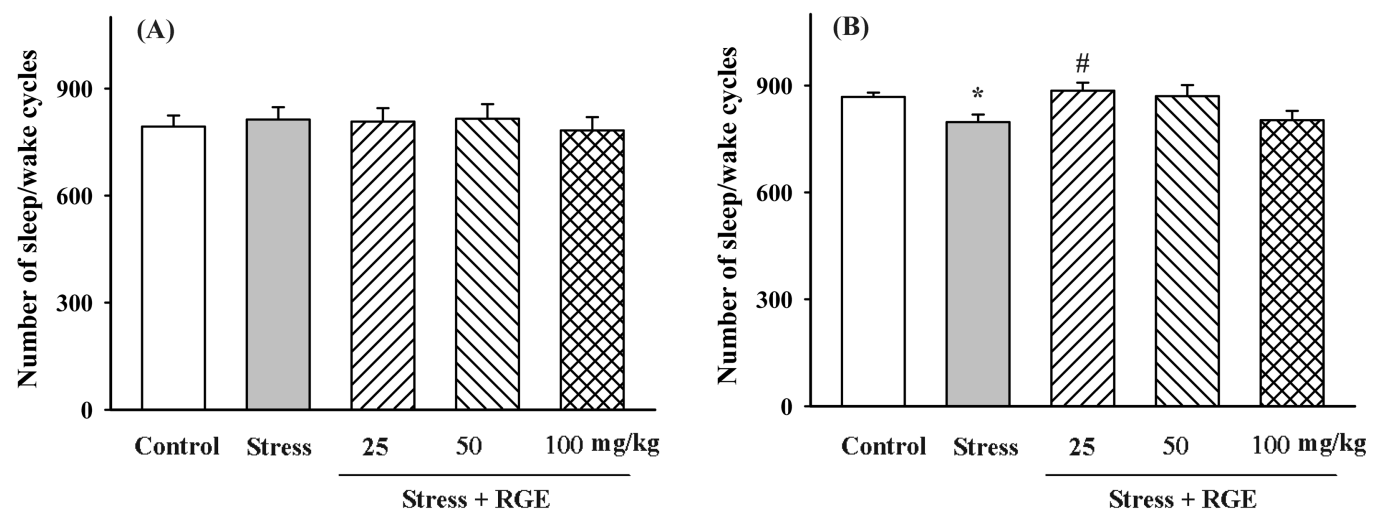

Fig. 2. The effect of RGE on number of sleep/wake cycles in acutely stressed rats. Numbers of sleep/wake change were evaluated. The data represent the mean \pm SE of sleep/wake change numbers. (A): Day time sleep/wake changes in acutely stressed rats. (B): Night time sleep/wake changes in acutely stressed rats. $* \mathrm{P}<0.05$, significantly different from control group; \#P<0.05, significantly different from stress group.

acutely stressed rats in night time recording $(\mathrm{F}(4,35)=$ $2.73, p<0.05)$. In the day time recording, the number of sleep-wake cycles was not changed by acute stress and RGE administration $(\mathrm{F}(4,35)=0.14, p=0.97)$. In the night time recording Post hoc comparisons revealed that the number of sleep-wake cycles was reduced in the acutely stressed rats. RGE $(25 \mathrm{~g} / \mathrm{kg})$ increased the number of sleep-wake cycles $(p<0.05$, Fig. 2A,B).

\section{Effects of RGE on EEG power density during NREM sleep of acutely stressed rats}

Fig. 3 shows the effect of RGE on NREM sleep EEG power density of acutely stressed rats. The ANOVAs were significant for comparisons in $\delta$-wave, $\theta$-wave and $\alpha$-wave power density of acutely stressed rats in day time record- ing $(\delta$-wave $[\mathrm{F}(4,35)=2.91, p<0.05] ; \theta$-wave $[\mathrm{F}(4,35)=$ $3.15, p<0.05]$; $\alpha$-wave $[\mathrm{F}(4,35)=2.93, p<0.05])$ and $\delta$ wave, $\theta$-wave power density during night time recording $(\delta$-wave $[\mathrm{F}(4,35)=2.98, p<0.05] ; \theta$-wave $[\mathrm{F}(4,35)=2.97$, $p<0.05])$. During NREM sleep, acutely stressed rats appeared to have increased $\delta$-wave, reduced $\theta$-wave, and similar $\alpha$-wave power density in the day time recording $(p<0.05$, Fig. 3A, B). At 25 and $50 \mathrm{mg} / \mathrm{kg}$, RGE increased the EEG power density of $\theta$-wave and $\alpha$-wave respectively ( $p<0.05$, Fig. 3A, B). Acute stress did not change the EEG power density in the night time NREM sleep. RGE at 25 and $50 \mathrm{mg} / \mathrm{kg}$ increased the $\delta$-wave and $\theta$-wave power density respectively $(p<0.05$, Fig. $3 \mathrm{~A}, \mathrm{~B})$. 

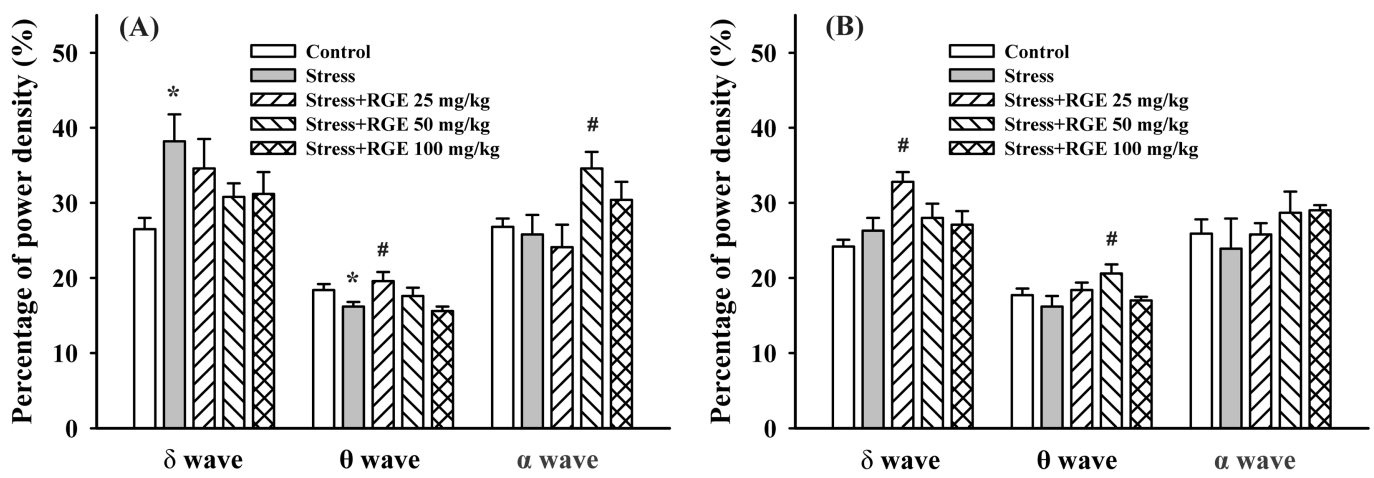

Fig. 3. The effect of RGE on EEG power density during NREM sleep in acutely stressed rats. EEG power density in $\delta$-wave, $\theta$-wave and $\alpha$-wave were evaluated. The data represent the mean \pm SE of EEG power density in 3 selected frequency bands in NREM sleep stage. (A): Day time EEG power density during NREM sleep in acute stressed rats. (B): Night time EEG power density during NREM sleep in acutely stressed rats. ${ }^{*} \mathrm{P}<0.05$, significantly different from control group; $\# \mathrm{P}<0.05$, significantly different from stress group.
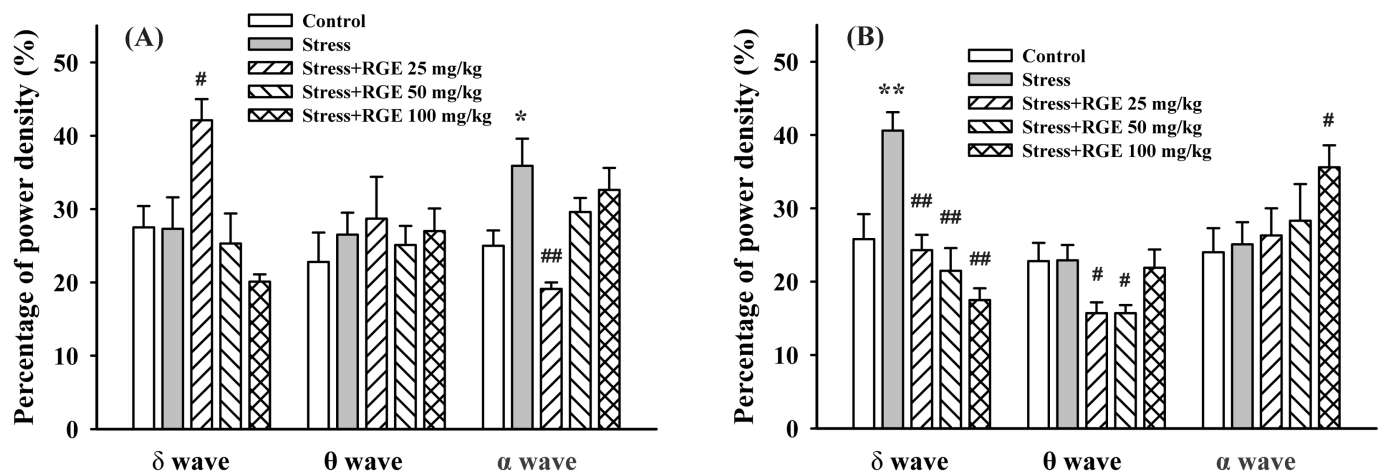

Fig. 4. The effect of RGE on EEG power density during REM sleep in acutely stressed rats. EEG power density in $\delta$-wave, $\theta$-wave and $\alpha$-wave were evaluated. The data represent the mean \pm SE of EEG power density in 3 selected frequency bands in REM sleep stage. (A): Day time EEG power density during REM sleep in acutely stressed rats. (B): Night time EEG power density during REM sleep in acutely stressed rats. $* \mathrm{P}<0.05$; $* * \mathrm{P}<0.01$, significantly different from control group; \#P<0.05; \#\#P<0.01, significantly different from stress group.

\section{Effects of RGE on EEG power density during REM} sleep of acutely stressed rats

Fig. 4 shows the effect of RGE on REM sleep EEG power density of acutely stressed rats. The ANOVAs were significant in $\delta$-wave, $\alpha$-wave power density of acutely stressed rats in day time recording ( $\delta$-wave $[\mathrm{F}(4,35)=3.55$, $p<0.05] ; \alpha$-wave $[\mathrm{F}(4,35)=3.93, p<0.01])$ and $\delta$-wave, $\theta$ wave, $\alpha$-wave power density during night time recording $(\delta$-wave $[\mathrm{F}(4,35)=3.98, p<0.01] ; \theta$-wave $[\mathrm{F}(4,35)=4.41$, $p<0.01] ; \alpha$-wave $[\mathrm{F}(4,35)=2.96, p<0.05])$. During REM sleep, acute stress increased day time $\alpha$-wave and night time $\delta$-wave power density $(p<0.05, p<0.01$, Fig. $4 \mathrm{~A}, \mathrm{~B})$. At a dose of $25 \mathrm{mg} / \mathrm{kg}$, RGE significantly increased day time $\delta$-wave and decreased $\alpha$-wave power density while at 25 and $50 \mathrm{mg} / \mathrm{kg}$, RGE could decrease night time $\delta$ - wave and $\theta$-wave power density $(p<0.05, p<0.01$, Fig. 4A, B). RGE at $100 \mathrm{mg} / \mathrm{kg}$ was also effective in decreasing $\delta$-wave but increased $\alpha$-wave power density in night time recording ( $p<0.05, p<0.01$, Fig. $4 \mathrm{~A}, \mathrm{~B})$.

Effects of RGE on EEG power density during wake time of acutely stressed rats

Fig. 5 shows the effect of RGE on EEG power density of wakefulness in acutely stressed rats. The ANOVAs were significant for comparisons across groups in $\delta$-wave power density of acutely stressed rats in day time recording $(\mathrm{F}(4,35)=4.98, p<0.01)$. During wakefulness, Post hoc comparisons revealed that acute stress increased the day time $\delta$-wave power density ( $p<0.01$, Fig. $5 \mathrm{~A}$ ), RGE at 25 $\mathrm{mg} / \mathrm{kg}$ reduced $\delta$-wave power density of day time in 

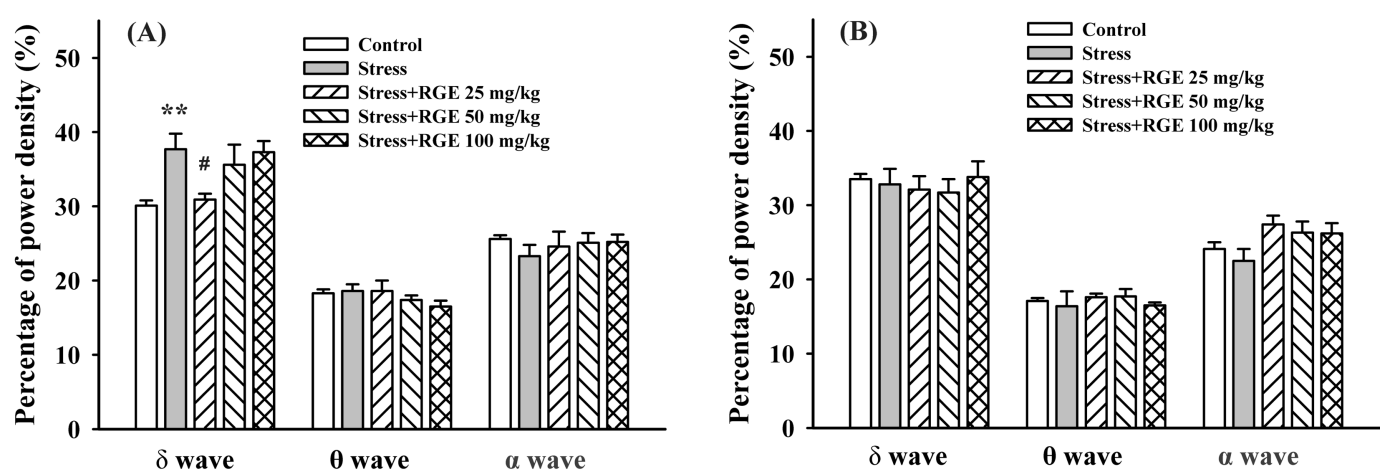

Fig. 5. The effect of RGE on EEG power density during wakefulness in acutely stressed rats. EEG power density in $\delta$-wave, $\theta$-wave and $\alpha$-wave were evaluated. The data represent the mean \pm SE of EEG power density in 3 selected frequency bands in the wakefulness stage. (A): Day time EEG power density during wakefulness in acute stressed rats. (B): Night time EEG power density during wakefulness in acutely stressed rats. ${ }^{*} \mathrm{P}<0.01$, significantly different from control group; \#P<0.05, significantly different from stress group.
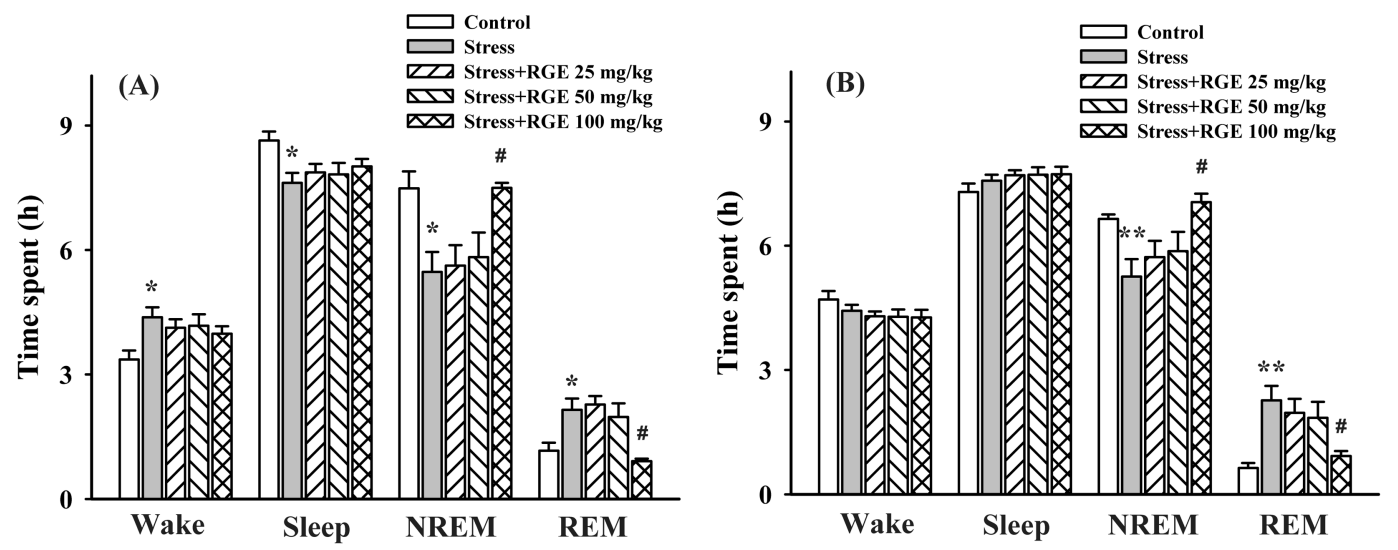

Fig. 6. The effect of RGE on sleep architecture in chronically stressed rats. The data represent the mean \pm SE of time spent in sleepwake state (Wake, Sleep (total sleep), NREM sleep, REM sleep) and the number of sleep/wake changes. (A): Day time sleep architecture in chronically stressed rats; (B): Night time sleep architecture in chronically stressed rats. $* \mathrm{P}<0.05 ; * * \mathrm{P}<0.01$, significantly different from control group; $\# \mathrm{P}<0.05$, significantly different from stress group.

acutely stressed rats $(p<0.05$, Fig. 5A). Acute stress and RGE did not induce any changes in the night time EEG power density of all the 3 selected frequency bands of wakefulness (Fig. 5B).

\section{Effects of RGE on sleep architecture of chronically} stressed rats

Fig. 6 shows the effect of RGE on the sleep architecture of chronically stressed rats. The ANOVAs were significant for comparisons across groups in time spent in wakefulness, total sleep, NREM and REM sleep during day time recording (Wakefulness $[\mathrm{F}(4,35)=2.74, p<0.05]$; Total sleep $[\mathrm{F}(4,35)=2.74, p<0.05]$; NREM sleep $[\mathrm{F}(4,35)=3.35$, $p<0.05]$; REM sleep $[\mathrm{F}(4,35)=3.60, p<0.05])$, but only NREM and REM sleep during night time recording
(Wakefulness $[\mathrm{F}(4,35)=1.04, p=0.40]$; Total sleep $[\mathrm{F}(4,35)=$ 1.04, $p=0.40]$; NREM sleep $[\mathrm{F}(4,35)=2.90, p<0.05]$; REM sleep $[\mathrm{F}(4,35)=4.81, p<0.01])$.

Post hoc comparisons revealed that chronic stress increased wakefulness, decreased total sleep in the day time recording without inducing any changes in the night time wakefulness and total sleep. Chronic stress increased REM and decreased NREM sleep in both the day and night time recording ( $p<0.05, p<0.01$, Fig. 6A, B). RGE, at 25,50 and $100 \mathrm{~g} / \mathrm{kg}$, did not change wakefulness and total sleep in chronic stressed rats, while RGE $100 \mathrm{~g} / \mathrm{kg}$ significantly ameliorated stress induced alterations in NREM and REM sleep in the day and night time sleep architecture ( $p £^{\circ} 0.05$, Fig. 6A,B). 
Effects of RGE on sleep/wake changes of chronically stressed rats

Fig. 7 presents the effect of RGE on sleep/wake changes in chronically stressed rats. The ANOVAs were significant for comparisons across groups in sleep/wake changes of chronically stressed rats in day time recording $(\mathrm{F}(4,35)=$ $2.92, p<0.05)$. Chronic stress increased the number of sleep-wake cycles only in the day time recording $(p<0.05$, Fig. 7A). RGE did not influence sleep/wake changes in the chronically stressed rats (Fig. 7A,B).

\section{Effects of RGE on EEG power density during NREM} sleep of chronically stressed rats

Fig. 8 shows the effect of RGE on NREM sleep EEG power density of chronically stressed rats. The ANOVAs were significant for comparisons in $\delta$-wave, $\theta$-wave power density during day time recording $(\delta$-wave $[\mathrm{F}(4,35)=2.94$, $p<0.05]$; $\theta$-wave $[\mathrm{F}(4,35)=4.74, p<0.01])$, and $\delta$-wave, $\alpha$ wave power density during nighttime recording ( $\delta$-wave $[\mathrm{F}(4,35)=2.90, p<0.05] ; \alpha$-wave $[\mathrm{F}(4,35)=2.99, p<0.05])$. Chronic stress increased the day time $\theta$-wave, but decreased the night time $\alpha$-wave power density of NREM sleep $(p<0.05, p<0.01$, Fig. 8A,B). Administration of a $25 \mathrm{mg} / \mathrm{kg}$ dose of RGE reduced day time $\delta$-wave power density while at $100 \mathrm{mg} / \mathrm{kg}$, RGE decreased the day time $\theta$-wave and increased the night time $\delta$-wave power density in NREM sleep $(p<0.05$, Fig. 8A, B).

\section{Effects of RGE on EEG power density during REM} sleep of chronically stressed rats

Fig. 9 shows the effect of RGE on REM sleep EEG power density of chronically stressed rats. The ANOVAs were significant for comparisons across groups in $\delta$-wave, $\theta$-wave power density during day time recording ( $\delta$-wave
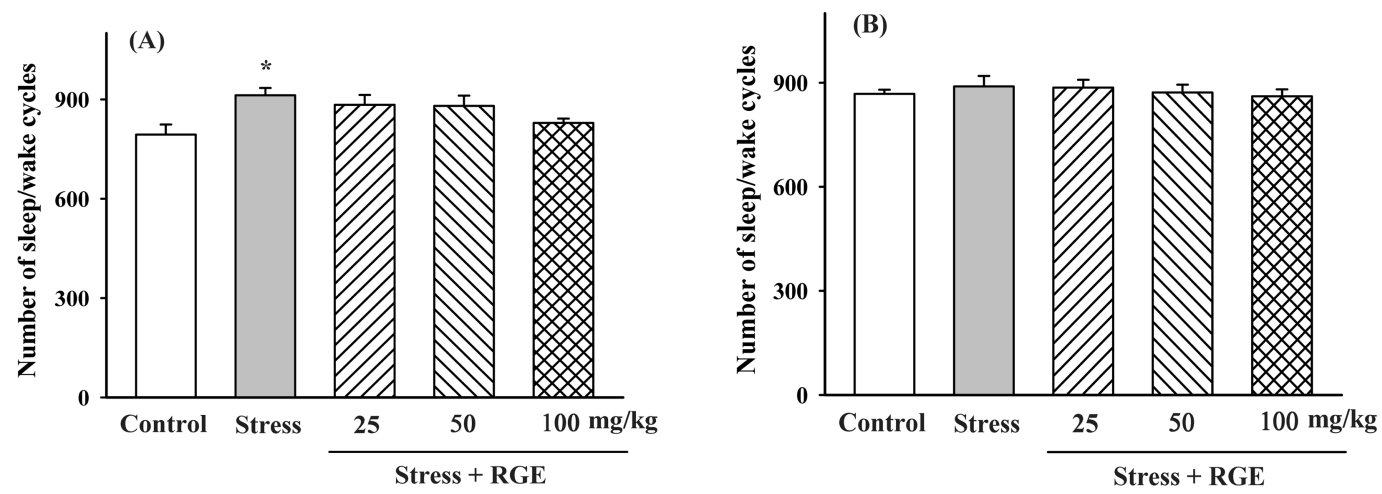

Fig. 7. The effect of RGE on number of sleep/wake cycles in chronically stressed rats. Numbers of sleep/wake change were evaluated. The data represent the mean \pm SE of sleep/wake change numbers. (A): Day time sleep/wake changes in chronically stressed rats. (B): Night time sleep/wake changes in chronically stressed rats. ${ }^{*} \mathrm{P}<0.05$, significantly different from control group.
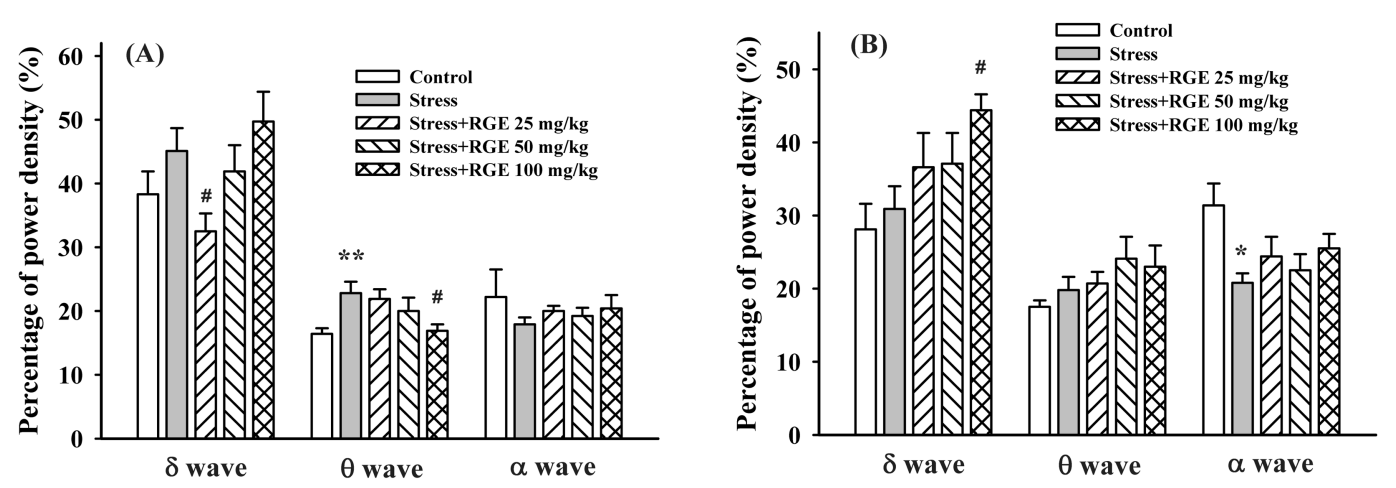

Fig. 8. The effect of RGE on EEG power density during NREM sleep in chronically stressed rats. EEG power density in $\delta$-wave, $\theta$ wave and $\alpha$-wave were evaluated. The data represent the mean \pm SE of EEG power density in 3 selected frequency bands in NREM sleep stage. (A): Day time EEG power density during NREM sleep in chronically stressed rats. (B): Night time EEG power density during NREM sleep in chronically stressed rats. $* \mathrm{P}<0.05 ; * * \mathrm{P}<0.01$, significantly different from control group; \#P<0.05, significantly different from stress group. 

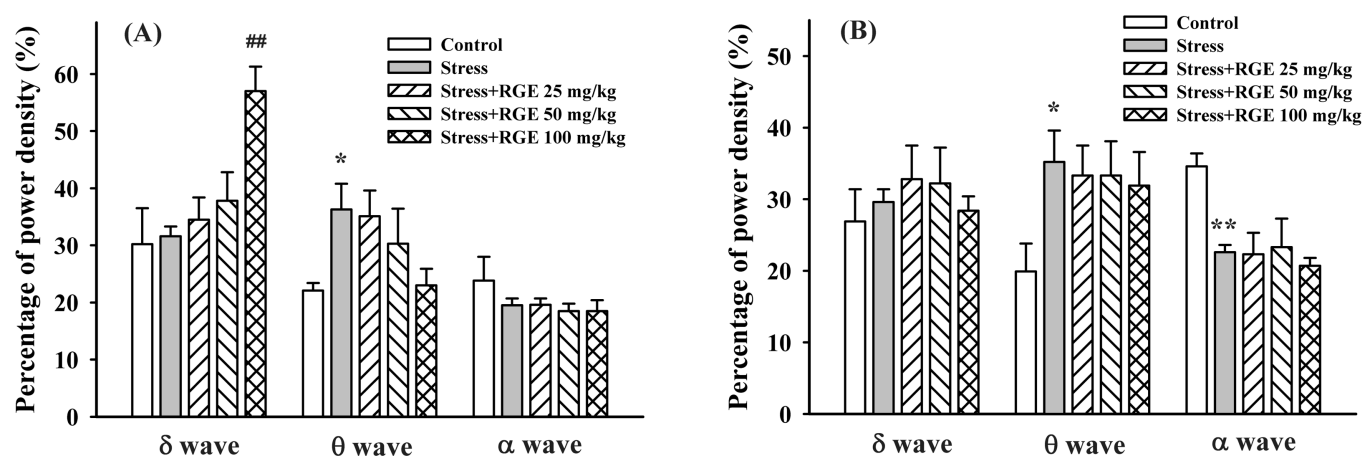

Fig. 9. The effect of RGE on EEG power density during REM sleep in chronically stressed rats. EEG power density in $\delta$-wave, $\theta$-wave and $\alpha$-wave were evaluated. The data represent the mean \pm SE of EEG power density in 3 selected frequency bands in REM sleep stage. (A): Day time EEG power density during REM sleep in chronically stressed rats. (B): Night time EEG power density during REM sleep in chronically stressed rats. ${ }^{*} \mathrm{P}<0.05$; $* * \mathrm{P}<0.01$, significantly different from control group; \#\#P<0.01, significantly different from stress group.
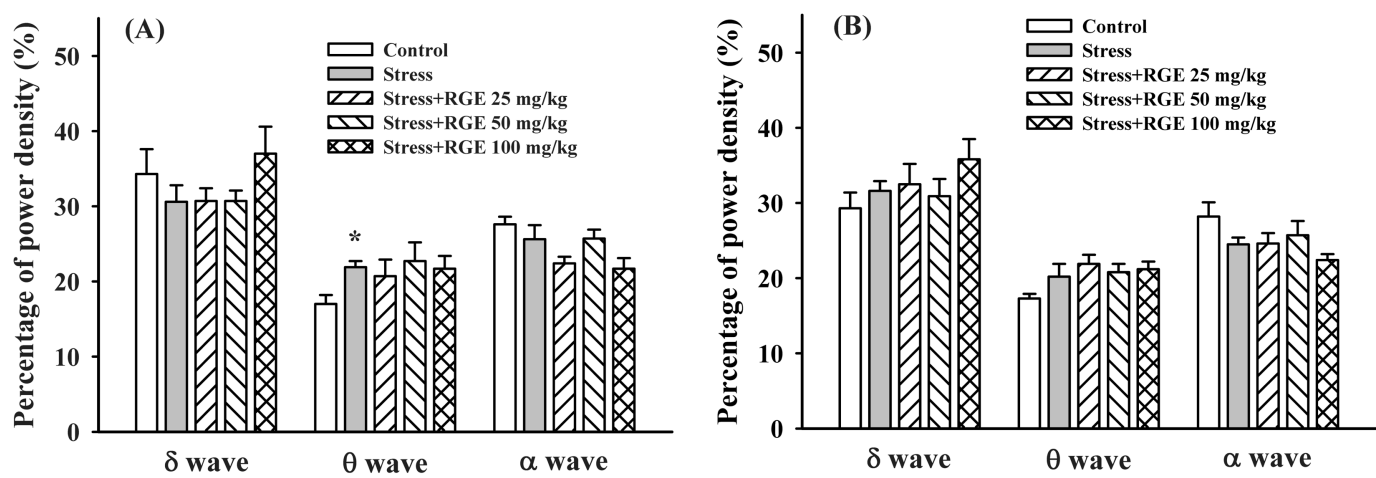

Fig. 10. The effect of RGE on EEG power density during wakefulness in chronically stressed rats. EEG power density in $\delta$-wave, $\theta$ wave and $\alpha$-wave were evaluated. The data represent the mean \pm SE of EEG power density in 3 selected frequency bands in the wakefulness stage. (A): Day time EEG power density during wakefulness in chronically stressed rats. (B): Night time EEG power density during wakefulness in chronically stressed rats. ${ }^{\mathrm{P}}<0.05$, significantly different from control group.

$[\mathrm{F}(4,35)=3.71, p<0.01] ; \theta$-wave $[\mathrm{F}(4,35)=3.45, p<0.05])$, and $\theta$-wave, $\alpha$-wave power density during night time recording $(\theta$-wave $[\mathrm{F}(4,35)=2.93, p<0.05]$; $\alpha$-wave $[\mathrm{F}(4,35)=$ $4.53, p<0.01])$. Chronic stress increased the è-wave power density in the day and night time recording and decreased the $\alpha$-wave power density only in the night time recording of REM sleep $(p<0.05, p<0.01$, Fig. 9A, B). RGE at 100 $\mathrm{mg} / \mathrm{kg}$ increased the day time $\delta$-wave power density in stressed rats ( $p<0.01$, Fig. 9A). The night time REM sleep EEG power density of the 3 selected frequency bands in chronically stressed rats was not changed by RGE $(p<0.05$, Fig. 9A, B).

Effects of RGE on EEG power density during wake time of chronically stressed rats

Fig. 10 shows the effect of RGE on EEG power density of wakefulness in chronically stressed rats. The ANOVAs were significant for comparisons across groups in $\theta$-wave power density during day time recording $(\mathrm{F}(4,35)=3.57$, $p<0.05)$. During wakefulness, Post hoc comparisons revealed that chronic stress increased the day time $\theta$-wave power density ( $p<0.05$, Fig. 10A). RGE at 25, 50 and 100 $\mathrm{mg} / \mathrm{kg}$ did not change the daytime EEG power density of chronically stressed rats. Chronic stress and RGE did not induce any changes in the night time EEG power density of all the 3 selected frequency bands of wakefulness (Fig. 10B).

\section{DISCUSSION}

In this study, we established an acute and chronic stress model by inducing restraint stress to rats. In this study, the 
wakefulness was decreased and the total sleep was significantly elevated in the acute restraint stressed condition, whereas the total and NREM sleep were significantly reduced in chronically stressed rat. By contrast, REM was significantly increased in the chronic restraint stressed condition. The cortical EEG activity also yielded significant differences in the acutely and chronically stressed group. Normally, stress-induced changes are self-limiting and adaptive unless events that override the "threshold" limits become irreversible and pathological [20]. Researchers found that there were different responses in sleep behaviors after acute and chronic stress [21, 22], and it is still not fully understood about the exact sleeping responses to different stressors in human and rodents, several factors, such as animal strain or age, different conditions in experimental process etc, might result in discrepancies between reports from different research groups. Our research have discrepancy with previous report in REM sleep changing after chronic stress [15], but our results agree with some other reports $[22,23]$, and suggest that the cortical EEG activity could reflect a stress condition.

Panax ginseng is used extensively for a wide variety of clinical ailments and to improve general physical and mental wellbeing [24]. People believe that ginseng possesses anti-stress and sleep stabilizing effects. Our investigation indicated that acute stressed rats with RGE administration exhibited an increase in wakefulness and a decrease in total, NREM, and REM sleep, particularly in the night time recording. In chronically stressed rats, RGE increased NREM sleep and decreased REM sleep; however, the time of wakefulness, total sleep and the number of sleep-wake cycle did not change. Our results are consistent with the belief that ginseng is effective in ameliorating stress-related sleep disorders and further support the idea that these effects are specifically focused on the balance between NREM and REM sleep [25].

Numerous reports have demonstrated that the modulation of total amount and the frequency of sleep depend on the specific activity patterns of the cortical EEG waves [26]. NREM sleep in experimental animals, also referred to as slow wave sleep (SWS), is known to be the deep sleep stage in humans and other mammals; it is defined by an oscillation of the EEG in the $\delta$-wave frequency range $(<2$ $\mathrm{Hz}$ in human, 0.75 to $4.0 \mathrm{~Hz}$ range in rats) [27]. EEG activities in the $\delta$-wave frequency range delineates NREM sleep and represent widespread, synchronized, firing patterns of cortical and thalamocortical neurons and reflect modulation of sleep [27, 28]. Sleep deprivation, or forced wakefulness, could induce a large increase in the ä-wave power [29]. This mechanism of sleep is thought to have an essential role for rest and maintenance of neural function. The regulation of the ä-wave activity in sleep is linked to synaptic potentiation and downscaling [29-31] with increases in the local $\delta$-wave activity in sleep after a motor learning task correlating with improved performance in humans [29, 32]. The power of the $\delta$-wave activity is a reliable parameter for an assessment of sleep depth and the regulation of sleep $[33,34]$. $\delta$-Wave activity relates to prior waking and is also considered to be a reliable indicator of time spent awake [35]. Studies have shown that the $\delta$-wave activity in NREM sleep typically declines in the course of the daily sleep period and increases in recovery sleep after a period of prolonged waking [26]. However, in our results, RGE decreased NREM and REM sleep that has decreased äwave activity. In particular, RGE decreased the night time REM sleep of acute stressed rats. Therefore, it is possible that RGE modulates the sleep architecture in a similar fashion to the physiological regulation that occurs during a nap or a period of excess sleep in the acute stress condition. In the chronic stress condition, a high dose of RGE (100 mg/kg) increased NREM and decreased REM sleep and was accompanied by an increase of $\delta$-wave activity during nighttime NREM sleep and daytime REM sleep. This indicates that $\delta$-wave activity is closely related to chronic stress induced sleep alterations and sleep modulation by RGE.

In this study, 25 and $50 \mathrm{mg} / \mathrm{kg}$ of RGE had similar effects on NREM and REM in acutely stressed rats, and had similar effects on the cortical EEG pattern, which was associated with decreased $\delta$-wave and $\theta$-wave activity in the night time REM sleep. One hundred $\mathrm{mg} / \mathrm{kg}$ of $\mathrm{RGE}$ could increase the power density of $\alpha$-wave activity on REM sleep without changing $\theta$-wave activity in night time REM sleep. In chronically stressed rats, only 100 $\mathrm{mg} / \mathrm{kg}$ RGE changed NREM and REM sleep, increased the EEG power density of $\delta$-wave in the nighttime NREM and REM sleep, and decreased $\theta$-wave activity in the daytime NREM sleep. These differences indicate that RGE is more effective at lower doses in modulating acute stress induced sleep disorders. Higher doses of RGE are needed to modulate chronic stress induced sleep disorders. These results suggest that the effects of RGE are more specifically related to the function of NREM and REM sleep. Our results also found that a low dose $(25 \mathrm{mg} / \mathrm{kg})$ and a high dose $(100 \mathrm{mg} / \mathrm{kg})$ of RGE showed different effects on $\delta$-wave power density in the wake process and REM sleep. This result provides an additional possible reason for the difference of sleep modulating effects between low 
and high dosage of RGE. REG extract may affect GABAergic systems, by interacting with ligand-bindings of $\mathrm{GABA}_{A}$ and $\mathrm{GABA}_{B}$ receptors [36]. Ginseng administration in animals has been shown behavioral changes which seem to be related to the regulation of GABAergic neurotransmission which play an important role in the action of CNS. Insomnia is one of the most frequent complaints encountered in a doctor's office and is most often related to anxiety and/or stress [37]. GABAergic modulation by ginseng is possibly involved in the alternation of sleep architecture and EEG power spectra in stressed animals. Further study is necessary to clarify the possible mechanisms.

It was widely accepted that ginseng has anti-stress effects, and its effects were closely related to modulation of hypothalamus-pituitary-adrenal axis function, decreasing corticosterone level in blood [38, 39]. Recent reports indicated that ginseng components could modulate normal sleep through GABAergic systems [40]. It could also reverse social isolation stress-induced decrease in pentobarbital sleep through $\mathrm{GABA}_{\mathrm{A}}$ receptor complex and neurosteroid function [41-43]. So it is possible that RGE ameliorated restraint stress-induced sleep disorder by its anti-stress effects in our study, but it might have several possible mechanisms, such as GABA and its transporter in brain, neurosteroid and its binding site on the $\mathrm{GABA}_{\mathrm{A}}$ receptor complex etc.

In summary, results of the current study have demonstrated that acute and chronic stresses induce sleep disorders with different patterns. RGE increased wakefulness and decreased the total, NREM and REM sleep in acute stressed rats; RGE also increase NREM but decreased REM sleep in chronically stressed rats. Regulation of the sleep architecture of RGE is focused on NREM and REM sleep, and involves modulation of $\alpha-, \theta$ - and $\delta$-waves activities in the cortical EEG. It is possible to discover novel active compounds from RGE which could modulate sleep through specific mechanisms. Further studies are needed to investigate the effects that specific RGE compounds have on the sleep architecture and EEG wave activity.

\section{ACKNOWLEDGEMENT}

This work was supported by Nonghyup Ginseng Central Research Institute and by a Grant from the Korean Ministry of Education, Science and Technology (The regional Core Research Program/Center for Healthcare Technology Development).

\section{REFERENCES}

1. Coon JT, Ernst E. Panax ginseng: a systematic review of adverse effects and drug interactions. Drug Safety 25: 323344 (2002)

2. Siegel RK. Ginseng abuse syndrome. Problems with the panacea. JAMA. 241: 1614-1615 (1979)

3. Cha HY, Seo JJ, Park JH, Choi KJ, Hong JT, Oh KW. Anxiolytic-like effects of total saponin fraction from Ginseng Radix Rubra on the elevated plus-maze model in mice. $\mathrm{J}$ Ginseng Res. 28: 132-135 (2004)

4. Ma Y, Eun JS, Oh KW. Therapeutic effects of ginseng on psychiatric disorders. J Ginseng Res. 31: 117-126 (2007)

5. Naval MV, Gomez-Serranilos MP, Carretero ME, Villar AM. Neuroprotective effects of a ginseng (Panax ginseng) root extract on astrocytes primary culture. J Ethnopharmacol. 112: 262-270 (2007)

6. Shah ZA, Gilani RA, Sharma P, Vohora SB. Cerebroprotective effect of Korean ginseng tea against global and focal models of ischemia in rats. J Ethnopharmacol. 101: 299-307 (2005)

7. Xiang YZ, Shang HC, Gao XM, Zhang BL. A comparison of the ancient use of ginseng in traditional Chinese medicine with modern pharmacological experiments and clinical trials. Phytotherpy Res. 22: 851-858 (2008)

8. Lee SP, Honda K, Rhee YH, Inoue S. Chronic intake of panax ginseng extract stabilizes sleep and wakefulness in food-deprived rats. Neurosci Lett. 111: 217-221 (1990)

9. Ma Y, Eun JS, Cheong JH, Rhee DK, Hong JT, Oh KW. Korea Red Ginseng alters electroencephalogram spectra of sleep-wake stage in rats. J Ginseng Res. 32: 220-225 (2008)

10. Ma Y, Kim YB, Nam SY, Cheong JH, Hong JT, Oh KW. The effect of Korea red ginseng on sleep architecture and electroencephalogram power spectra in rats. Sleep \& Biol Rhythms. 7: 78-83 (2009)

11. Bonnet C, Marinesco S, Debilly G, Kovalzon V, Cespuglio R Influence of a 1-h immobilization stress on sleep and CLIP [ACTH(18-39)] brain contents in adrenalectomized rats. Brain Res. 853: 323-329 (2000)

12. Fulder S. Ginseng and the hypothalamic-pituitary control of stress. Am J Chinese Med. 9: 112-118 (1981)

13. Gonzalez MM, Debilly G, Valatx JL, Jouvet M. Sleep increase after immobilization stress: Role of the noradrenergic locus coeruleus system in the rat. Neurosci Lett. 202: 5-8 (1995)

14. Vazquez-Palacios G., Retana-Marquez S, Bonilla-Jaime H, Velazquez-Moctezuma J. Stress-induced REM sleep increase is antagonized by naltrexone in rats. Psychopharmacol. 171: 186-190 (2004)

15. Kant G, Pastel RH, Bauman RA, Meininger G.R, Maughan KR, Robinson TN. 3rd, Wright WL, Covington PS. Effects 
of chronic stress on sleep in rats. Physiol \& Behav. 57: 359365 (1995)

16. Rampin C, Cespuglio R, Chastrette N, Jouvet M. Immobilisation stress induces a paradoxical sleep rebound in rat. Neurosci Lett. 126: 113-118 (1991)

17. Sanford LD, Yang L, Liu X, Tang X. Effects of tetrodotoxin (TTX) inactivation of the central nucleus of the amygdala (CNA) on dark period sleep and activity. Brain Res. 1084: 80-88 (2006)

18. Durán P, Galler JR, Cintra L, Tonkiss J. Prenatal malnutrition and sleep states in adult rats: effects of restraint stress. Physiol Behav. 89: 156-163 (2006)

19. Goebel M, Stengel A, Wang L, Taché Y. Restraint stress activates nesfatin-1-immunoreactive brain nuclei in rats. Brain Res. 1300: 114-124 (2009)

20. Schurr A. Energy metabolism, stress hormones and neural recovery from cerebral ischemia/hypoxia. Neurochem Int. 41: 1-8 (2002)

21. Gilad G.M, Gilad V.H. Stress-induced dynamic changes in mouse brain polyamines: Role in behavioral reactivity. Brain Res. 943: 23-29 (2002)

22. Hayashi Y, Tanaka J, Morizumi Y, Kitamura Y, Hattori Y. Polyamine levels in brain and plasma after acute restraint or water-immersion restraint stress in mice. Neurosci Lett. 355: 57-60 (2004)

23. Sohn HS, Park YN, Lee SR. Effect of immobilization stress on brain polyamine levels in spontaneously hypertensive and Wistar-Kyoto rats. Brain Res Bull. 57: 575-579 (2002)

24. Bahrke MS, Morgan WR. Evaluation of the ergogenic properties of ginseng: an update. Sports Med. 29: 113-133 (2000)

25. Rhee YH, Lee SP, Honda K, Inoue S. Panax ginseng extract modulates sleep in unrestrained rats. Psychopharmacol. 101: 486-488 (1990)

26. Franken P, Chollet D, Tafti M. The homeostatic regulation of sleep need is under genetic control. J Neurosci. 21: 26102621 (2001)

27. Maret S, Franken P, Dauvilliers Y, Ghyselinck NB, Chambon P, Tafti M. Retinoic acid signaling affects cortical synchrony during sleep. Science 310: 111-113 (2005)

28. Steriade M, McCormick DA, Sejnowski TJ. Thalamocortical oscillations in the sleeping and aroused brain. Science 262: 679-685 (1993)

29. Kitaoka K, Hattori A, Chikahisa S, Miyamoto K, Nakaya Y,
Sei H. Vitamin A deficiency induces a decrease in EEG delta power during sleep in mice. Brain Res. 1150: 121-130 (2007)

30. Tononi G, Cirelli C. Sleep and synaptic homeostasis: a hypothesis. Brain Res. Bull. 62: 143-150 (2003)

31. Tononi G, Cirelli C. Sleep function and synaptic homeostasis. Sleep Med Rev. 10: 49-62 (2006)

32. Huber R, Ghilardi MF, Massimini M, Tononi G.. Local sleep and learning. Nature 430: 78-81 (2004)

33. Borbely AA. From slow waves to sleep homeostasis: new perspectives. Arch Ital De Biol. 139: 53-61 (2001)

34. Borbely AA, Achermann P. Sleep homeostasis and models of sleep regulation. J Biol Rhythms. 14: 557-568 (1999)

35. Deboer T, Tobler I. Slow waves in the sleep electroencephalogram after daily torpor are homeostatically regulated. Neuroreport 11: 881-885 (2000)

36. Kimura T, Saunders PA, Kim HS, Rheu HM, Oh KW, Ho IK. Interactions of ginsenosides with ligand-bindings of GABA(A) and GABA(B) receptors. Gen Pharmacol. 25: 193-199 (1994)

37. Gottesmann C. The transition from slow-wave sleep to paradoxical sleep: evolving facts and concepts of the neurophysiological processes underlying the intermediate stage of sleep. Neurosci \& Biobehavioral Rev. 20: 367-387 (1996)

38. Kita T, Hata T, Kawashima Y, Kaku T, Itoh E. Pharmacological actions of ginseng saponin in stressed mice. J Pharmacobiodyn. 4: 381-393 (1981)

39. Takagi K, Saito H, Tsuchiya M. Pharmacological studies of Panax ginseng root: pharmacological properties of a crude saponin fraction. Jpn J Pharmacol. 22: 339-346 (1972)

40. Kitaoka K, Uchida K, Okamoto N, Chikahisa S, Miyazaki T, Takeda E, Séi H. Fermented ginseng improves the first-night effect in humans. Sleep 32: 413-421 (2009)

41. Huong NT, Matsumoto K, Watanabe H. The antistress effect of majonoside- $\mathrm{R}_{2}$, a major saponin component of Vietnamese ginseng: neuronal mechanisms of action. Methods Find Exp Clin Pharmacol. 20: 65-76 (1998)

42. Nguyen TT, Matsumoto K, Yamasaki K, Watanabe H. Majonoside- $\mathrm{R}_{2}$ reverses social isolation stress-induced decrease in pentobarbital sleep in mice: possible involvement of neuroactive steroids. Life Sci. 61: 395-402 (1997)

43. Nguyen TT, Matsumoto K, Yamasaki K, Nguyen MD, Nguyen TN, Watanabe $H$. Effects of majonoside- $R_{2}$ on pentobarbital sleep and gastric lesion in psychologically stressed mice. Pharmacol Biochem Behav. 53: 957-963 (1996) 